\title{
Crucial Stem Cell Experiments? \\ Stem Cells, Uncertainty, and Single-Cell Experiments*
}

\author{
Melinda Bonnie FAGAN
}

Received: 01/09/2014

Final Version: 15/03/2015

BIBLID 0495-4548(2015)30:2p.183-205

DOI: $10.1387 /$ theoria. 12707

ABSTRACT: I have previously argued that stem cell experiments cannot demonstrate that a single cell is a stem cell (Fagan 2013a, b). Laplane and others dispute this claim, citing experiments that identify stem cells at the singlecell level. This paper rebuts the counterexample, arguing that the alleged 'crucial stem cell experiments' do not measure self-renewal for a single cell, do not establish a single cell's differentiation potential, and, if interpreted as providing results about single cells, fall into epistemic circularity. I then discuss the source of the dispute, locating it in differences between philosophical and experimental perspectives.

Keywords: experiment, stem cells, models, crucial experiments, in vivo/in vitro.

RESUMEN: He defendido que los experimentos con células madre no pueden mostrar que una sola célula es una célula madre (Fagan 2013a, b). Laplan, entre otros, cuestionan esta afirmación apelando a experimentos que identifican células madre a nivel unicelular. Este artículo refuta el contraejemplo argumentando que los supuestos "experimentos cruciales con células madre" no miden la autorrenovación de una única célula, que no establecen el potencial de diferenciación de una célula, y que, si se interpretan como si aportaran resultados sobre células únicas, incurren en una circularidad epistémica. A continuación discutiré la fuente de esta disputa localizándola en las diferencias entre la perspectiva filosófica y la experimental.

Palabras clave: Experimento, células madre, modelos, experimentos cruciales, in vivo/in vitro.

\section{Introduction}

Stem cells are much-discussed but elusive scientific entities. They have received widespread attention since 1998, when human embryonic stem cells were first experimentally produced (Thomson et al 1998). A subsequent milestone is 'reprogramming' of mature mammalian cells into stem cells (2006-2007), for which Shinya Yamanaka received the 2012 Nobel Prize in Physiology or Medicine (Takahashi and Yamanaka 2006, Takahashi et al 2007, Nobel Committee 2012). ${ }^{1}$ Even these briefly-sketched examples highlight an important feature of stem cell research: stem cells are characterized in terms of their origins

* This paper has benefited from comments and discussion with Allan Franklin, Lucie Laplane, PSX4 participants, PSA 2012 participants, an anonymous reviewer for Cell Stem Cell, and anonymous reviewers for THEORIA - An International Journal for Theory, History and foundations of Science. The research was funded by a Rice University Faculty Innovation Fellowship (HRC).

1 This prize was shared with John Gurdon, who fifty years earlier successfully reprogrammed frog nuclei using egg cytoplasm. 
and outcomes, not properties of the cells themselves. That is, stem cells are understood indirectly, in terms of where they come from and what they might do. Nearly all humanities and social science literature on the subject is concerned with what biological sources can be used to produce stem cells (e.g., human embryos, eggs, or mature tissues). ${ }^{2}$ Stem cell research is motivated by the hope of treating a wide range of pathologies for which current therapies are inadequate, including spinal injury, neurodegenerative diseases, heart attack and diabetes. So the biological sources and therapeutic results of stem cell research are wellcharacterized. While debate about the former has settled into a pattern of apparently intractable disagreement (Thompson 2013), attainment of the latter hinges on experiments that reveal and bring under our control the properties of stem cells themselves - molecular, cellular, and developmental.

This paper builds on earlier work, examining stem cell experiments from a philosophy of science perspective (Fagan 2013a, b, forthcoming). A disagreement in this nascent philosophical literature brings out important contrasts between this perspective and that of experimental biologists. Philosophy has long prioritized theory over experiment, with the consequence that experiment-driven fields like stem cell research have received very little attention from philosophers of science. My own approach aims to address this lacuna, by deploying tools from recent philosophy of science that are well-suited to engage with experimental practices: accounts of models and modeling, mechanistic explanation, and causal intervention (Fagan 2013a). However, even so, there are subtle differences between my philosophical approach and that of stem cell researchers. This paper sets out the debate, defends my original position, and investigates the source of dispute by explicating background assumptions that distinguish my view from that of experimentalists. These reflections on the relationship between philosophical and experimental inquiry may extend to other projects in philosophy of experiment.

First, some background on stem cell biology and its central concept: the stem cell. Stem cells, though incompletely understood, are not wholly mysterious entities. Their general scientific definition is in terms of developmental capacities:

\footnotetext{
"Stem cells are defined as having the capacity to both self-renew and give rise to differentiated cells" (Ramelho-Santos and Willenbring 2007, 35). ${ }^{3}$
}

Cells reproduce by binary division; in a division event, a parent cell divides to yield two offspring cells. ${ }^{4}$ Self-renewal is production of offspring that resemble the parent cell. Differentiated cells are specialized to play particular roles within a healthy organism, making up its diverse organs and tissues: nerves, blood, bone, muscle, etc. So stem cells are cells capable of producing both cells that resemble the parent 'stem' and cells that are more specialized

2 E.g., Maienschein 2003, Gottweis et al 2009, Mazzolini and Rheinberger 2012, Thompson 2013.

3 Other general definitions add qualifications to this basic characterization; e.g., Essentials of Stem Cell Biology (Melton and Cowan 2009, xxiv) and information pages on the websites of the US National Institutes of Health (http://stemcells.nih.gov/info/basics/basics1.asp) and the European Stem Cell Network (http://www.eurostemcell.org/stem-cell-glossary). The basic definition above is robust, however; a common core of all the general definitions on offer.

4 There are two modes of cell division: mitosis and meiosis. Stem cell phenomena involve mitosis; the term "cell division" here refers to that mode only. 
than the parent. ${ }^{5}$ Self-renewal and differentiation are relational processes, involving comparison across cell generations. Differentiation further presupposes a directed developmental axis along which cells can be classified as more or less specialized. This general definition of 'stem cell' can be explicated more precisely by recasting it as a simple abstract model with four variables: cell lineage $(L)$, cell divisions/time interval $(n)$, characters of candidate stem cells $(C)$, and mature cell characters $(M){ }^{6}$ This explication of the stem cell concept highlights the question of how the model connects to biological reality: the cellular phenomena of interest to stem cell researchers.

Briefly, values of the model's variables are specified by experiments that aim to identify and characterize stem cells. That is, it is by experiment that the model is linked to biological entities that can be correctly termed 'stem cells.' These experiments, in practice, share a basic design. A consequence of the model and the experimental design, taken together, is that stem cells cannot be experimentally identified at the single-cell level (Fagan 2013a, 2013b).

Opposing this thesis, philosopher Lucie Laplane and several stem cell researchers have argued that some experiments can and do identify stem cells at the single-cell level (Laplane 2013, pers. comm.). These allegedly 'crucial stem cell experiments' involve transplanting a single cell into an animal (an inbred mouse), and then tracking that cell's descendants over time. If the result is long-term maintenance of a tissue derived from the transplant, then (they argue) we know that self-renewal and differentiation have occurred. Self-renewal must occur, because individual cells have short lifespans, yet the tissue is maintained for months or years. Differentiation must occur, because the specialized tissue cells are descended from the transplanted cell. Such an experiment therefore provides unequivocal evidence that the single transplanted cell is a stem cell. So 'crucial stem cell experiments' appear to flatly contradict an important consequence of my modeling approach, demonstrating that some experiments can unequivocally identify single stem cells. The following sections examine this debate, defending my original claim of stem cell uncertainty while also doing justice to the intuitions that make the counterexample compelling from an experimental standpoint. I then argue that these intuitions can be reconciled with my modelbased approach.

The paper is organized as follows. The next section sets out my model-based approach, and shows how the evidential constraint noted above follows from this and the basic design of stem cell experiments. Section 3 presents Laplane's proposed counterexample in detail, beginning with a recent scientific illustration and from this extracting the key features of crucial stem cell experiments. I next argue that this example does not contradict my claim that single stem cells cannot be experimentally identified, for at least three reasons: the allegedly crucial experiments do not measure self-renewal for a single cell (Section 4a), do not establish a single cell's differentiation potential (Section 4b), and, if their results are interpreted in terms of single cells, commit an epistemic circularity (Section 4c). The rest of the paper delves into sources of the dispute, articulating an important contrast between my perspective and that of experimental scientists. Section 5 shows how the model-based ap-

\footnotetext{
5 It has often been noted that the general definition of 'stem cell' is inadequate as a definition in the traditional philosophical sense of providing necessary and sufficient conditions for correct application of a term. But rather than attempt an improved analysis, I treat the general definition of 'stem cell' as indicating a concept that can be further explicated as an abstract model (see main text).

6 This model is presented in more detail below (Section 2).
} 
proach accommodates the allegedly crucial stem cell experiments, enrolling them as support rather than disconfirmation. Section 6 explicates several assumptions of experimentalists, which undercut or obscure the model-based perspective. I conclude by reflecting on some distinctive challenges for philosophy of experiment, suggested by this case.

\section{Stem cell uncertainty ${ }^{7}$}

My model-based approach explicates the general definition of stem cells as an abstract model, in Giere's sense $(1988,2004)$. An abstract model, on this view, consists of objects and relations organized so as to be similar to a target system in certain respects and to certain degrees, with standards determined according to model-users' purpose. ${ }^{8}$ More succinctly, " $S$ uses [model] $X$ to represent $W$ [an aspect of the real world] for purposes $P$ " (Giere 2004, 743). The purpose of this model is to represent the stem cell concept in as few terms as possible, while making explicit the assumptions involved. It is in this sense a minimal model, designed to represent a scientific concept rather than biological entities directly. The model presupposes several core tenets of cell biology: cells reproduce by binary division (see above); a cell's life begins with a division event and ends with either a second division event yielding two offspring or cell death and no offspring; and generations of cells linked by reproductive division form a lineage. The simplest possible lineage $L$ is produced by one cell division event, forming a genealogical structure consisting of two cell generations. Generalizing from this case brings in another variable: $n$, the number of cell divisions or 'cycles."

In the simple abstract model, both self-renewal and differentiation occur within a cell lineage $L$ involving $n$ divisions. In the limiting case of $n=1$, self-renewal and differentiation occur in $L$ just in case one offspring cell is similar to the parent, while the other is more specialized. However, no two cells are similar or different in every respect. The comparative concepts of self-renewal and differentiation should be understood as relative to a set of variable characters, values of which are compared across generations. For self-renewal, these characters are properties of individual cells, such as size, shape, surface expression level of a receptor, or internal concentration of a protein. Because there is no agreed-upon list of the characters relevant for stem cell self-renewal, the set of these characters is another variable, $C$. These three variables $(L, C$, and $n)$ suffice to define self-renewal: self-renewal occurs in lineage $L$ just in case parent and offspring cells in $L$ have the same values of character set $C$ for time interval (number of cell cycles) $n$.

Differentiation is more complicated, because this process presupposes an axis of increasing specialization on which character-values can be ordered. The endpoint of this axis is the 'terminus' of cell development: character-values of a mature, specialized cell. Each mature

7 This section summarizes material that is treated at greater length in Fagan 2013a. See also Fagan 2013b, forthcoming.

${ }^{8}$ Giere's 'similarity' account of the model-target relation has been criticized, and a number of alternatives proposed. The arguments here are independent of these ongoing debates (see Fagan 2013a, Chapter 2, for further discussion).

9 In the simplest case of a cell lineage, $n=1$. If an estimate of cell division rate is available for cells of lineage $L$, the value of $n$ can be converted to an interval of calendar time, ranging in practice from hours to decades. 
cell type is distinguished from others by particular values for a set of morphological, biochemical, and molecular characters. These mature cell characters include size, shape, pattern of gene expression, and physiologically significant behavior (e.g., contraction, antibody production, electrochemical signaling). So the set of characters relevant for differentiation are those with values that distinguish the mature cell type(s) of interest (variable $M$ ). Differentiation in lineage $L$ is defined in terms of $\mathrm{M}_{\mathrm{i}}$ (character-values of mature cells of one or more types) and a time-interval $\left(t_{1}-t_{2}\right)$. A cell differentiates over some time interval just in case its character-values for $M$ are more similar to those of mature cells $\left(\mathrm{M}_{\mathrm{i}}\right)$ at the end of that interval $\left(t_{2}\right)$ than the beginning $\left(t_{1}\right) \cdot{ }^{10}$ Variables $n$ and $t_{1}-t_{2}$ are inter-convertible if an estimate of cell division rate is available $\left(n\right.$ multiplied by cell division rate $=$ time interval $\left.t_{1}-t_{2}\right)$.

The abstract stem cell model combines self-renewal and differentiation processes, defined as stated above, into a single lineage $L$. The abstract model is a structure of objects ('cells') with characters $C$ and $M$, the values of which vary over $n$ or $\mathrm{t}_{1}-\mathrm{t}_{2}$ so as to satisfy the above definitions of self-renewal and differentiation (Figure 1). A stem cell, in this model, is the origin of a cell lineage $L$, with maximal self-renewal and differentiation capacity relative to a time interval of interest $(n)$ and two character-sets for comparison across cell generations: $C$ (characters of candidate stem cells) and $M$ (mature cell characters). This model is abstract not only in the sense that it is non-material, but also in that it represents the general stem cell concept, rather than straightforwardly corresponding to any concrete biological entities. Further representational assumptions are needed to connect elements of the model to biological targets. Cells in the model can be interpreted, for example, as single-

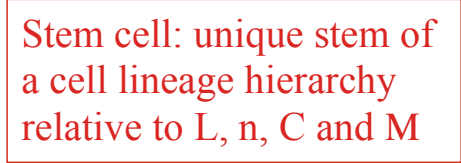

Cell lineage $L$

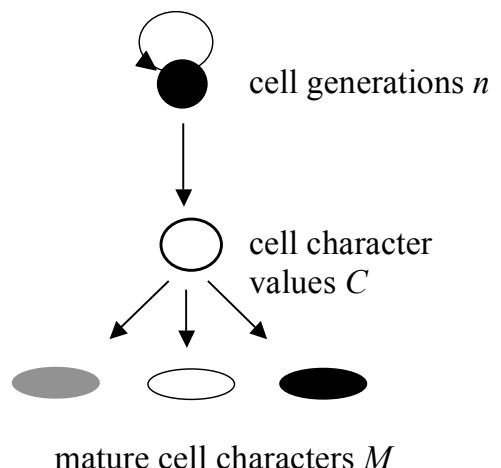

mature cell characters $M$

Figure 1. Abstract stem cell model

10 Note that this definition is inclusive, and does not distinguish between robust differentiation processes and transient fluctuations in cell characters due to stochastic noise in gene expression. The latter are not considered differentiation in scientific practice, and so this account of differentiation requires refinement to be fully integrated with current stem cell research. One way to accomplish this would be to limit values of $t_{2}-t_{1}$ to intervals indicative of robust differentiation processes. However, continued controversy about the nature of stem cells means that the issue needs careful examination before such constraints can be imposed. This examination is a task for another paper. Thanks to an anonymous reviewer for THEORIA for bringing up this point. 
cells, cell populations, or cell types. This paper is concerned only with the first. ${ }^{11}$ Moreover, to represent any particular biological phenomenon, the values of variables $L, n, C, M$ must be specified. These specifications are made by experimental methods, to which I now turn.

Stem cell research encompasses a wide variety of experimental techniques and designs. My concern here is only with one kind of experiment: that which aims to identify and characterize stem cells in accordance with the abstract model. Experiments with this kind are crucial in linking the abstract stem cell concept to concrete biological phenomena. The original experimental identification and characterization of all the major varieties of stem cell (e.g., mESC, hESC, HSC, iPSC, etc.) follows a common pattern, which I outline below. For ease of exposition, I refer to these as 'stem cell experiments,' although they are far from the only kind performed using stem cells. They have special significance, however, as the experiments by which stem cells are identified and characterized as such. It is via these experiments (and no others, in current practice) that we learn what biological entities conform to the abstract stem cell model. Stem cell experiments are the class of experiments that provide evidence that certain cells are, or are not, stem cells.

These experiments exhibit a basic structure of three stages (Figure 2). The starting point is a multicellular organism, the source of cells that specifies lineage $L$. A lineage $L$ of cells of a multicellular organism is further specified by species, age, and location within the source organism. For example, human embryonic stem cells are derived from the inner cell mass of very early ( $~ 5 \mathrm{~d}$ post-fertilization) human embryos. ${ }^{12}$ Returning to the general class
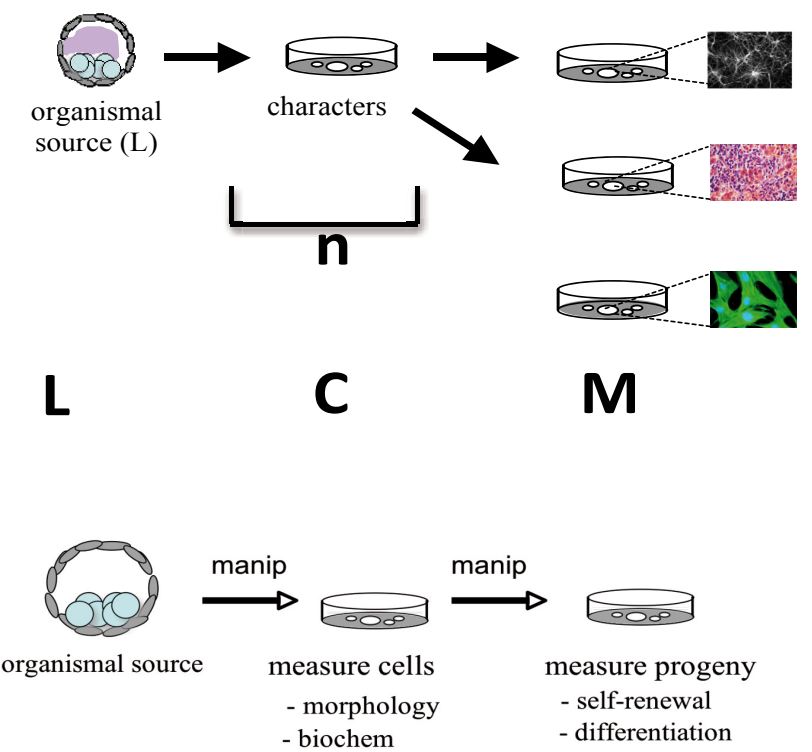

Figure 2. Stem cell experiments aim to show that biological cells have maximal self-renewal and differentiation potential, relative to specification of variables $L N, C, M$

11 For discussion of the latter two representational assumptions, see Fagan 2013a.

12 For more detail about how various types of stem cells are identified by experiments of this design, see Fagan 2013a, Chapter 2. 
of stem cell experiments: from the organismal source, cells are extracted and placed in a new environment, in which candidate stem cell characters are measured $(C)$. Often but not always, self-renewal is measured at this stage (specifying $n$ for self-renewal). The measured cells are then moved to another environment, in which capacities for differentiation can be realized. Finally, characters of differentiated cells are measured $(M)$; the duration of this part of the experiment also specifies $t_{1}-t_{2}$ (or $n$ ) for differentiation. Overall, stem cell experiments consist of two manipulations, each followed by measurement, of cells from some organismal source.

Experiments with this design can show that biological cells have maximal self-renewal and differentiation potential for certain values of $L, n, C, M$. Lineage $L$ is specified by the source organism and tissue from which cells are extracted, time interval $n$ by the duration of the experiment, and cell characters $C$ and $M$ by the cell properties measured during the experiment. Different experiments within the broader class of stem cell experiments specify different values of one or more of the model's variables, and thereby identify different kinds of stem cell. The same biological cells can qualify as stem cells on some assignments of values to the model's variables, and not others. This result makes sense of the rather bewildering diversity of stem cells in scientific practice (Table 1), which all conform to the general definition yet exhibit different morphologies, developmental capacities, and molecular characters. One important consequence of this model-based account is that substantive claims about stem cells should be understood in terms of experimental methods used to identify them. This guideline cuts short fruitless debates between groups using different experimental methods to identify stem cells of interest.

\section{Table 1. Model-based classification of stem cells}

\begin{tabular}{|l|l|l|l|l|}
\hline Type & \multicolumn{1}{|c|}{ L (source) } & \multicolumn{1}{|c|}{ C } & \multicolumn{1}{|c|}{ M } \\
\hline ESC & 5d embryo ICM & $\begin{array}{l}\text { cell size, cell shape, gene } \\
\text { expression, karyotype, } \\
\text { telomerase activity, alk-phos, } \\
\text { cell surface molecules }\end{array}$ & $\geq 50$ divs & $\begin{array}{l}\text { traits of cells from } \\
\text { three germ layers }\end{array}$ \\
\hline HSC & $\begin{array}{l}\text { BM, cord, peripheral } \\
\text { blood }\end{array}$ & $\begin{array}{l}\text { cell size, density, light scatter, } \\
\text { surface molecules, cell cycle } \\
\text { status }\end{array}$ & $\begin{array}{l}>6 \\
\text { months }\end{array}$ & $\begin{array}{l}\text { traits of main } \\
\text { blood and } \\
\text { immune cell } \\
\text { lineages }\end{array}$ \\
\hline NSC & $\begin{array}{l}\text { basal lamina of } \\
\text { ventricular zone }\end{array}$ & $\begin{array}{l}\text { cell morphology, surface } \\
\text { markers, gene expression, } \\
\text { cytokine response }\end{array}$ & $\begin{array}{l}\text { months to } \\
\text { years }\end{array}$ & $\begin{array}{l}\text { traits of neurons, } \\
\text { astrocytes, and } \\
\text { oligodendrocytes }\end{array}$ \\
\hline iPSC & $\begin{array}{l}\text { various (relatively } \\
\text { mature cells) }\end{array}$ & $\begin{array}{l}\text { colony shape, cell size, cell } \\
\text { shape, nucleus/cytoplasm } \\
\text { ratio, cell surface molecules, } \\
\text { activity and expression of } \\
\text { specific proteins, gene } \\
\text { expression (specific and } \\
\text { global), histone modifications } \\
\text { at key locations }\end{array}$ & 250 divs & $\begin{array}{l}\text { traits of cells from } \\
\text { three germ layers }\end{array}$ \\
\hline GSC & 5-9wk gonadal ridge & $\begin{array}{l}\text { colony shape, alk-phos, surface } \\
\text { expression (SSEA-1, SSEA-3, } \\
\text { SSEA-4, TRA-1-60,TRA-1-81) }\end{array}$ & 20-25 wks & $\begin{array}{l}\text { traits of cells from } \\
\text { three germ layers }\end{array}$ \\
\hline EC & teratocarcinoma (129) & $\begin{array}{l}\text { cell shape, morphology, } \\
\text { production of embryoid bodies, } \\
\text { surface molecules, enzymes }\end{array}$ & unlimited & $\begin{array}{l}\text { traits of cells from } \\
\text { three germ layers, } \\
\text { teratocarcinoma }\end{array}$ \\
\hline
\end{tabular}


A second consequence is an evidential constraint on experimental measurement of self-renewal and differentiation potential. The constraint arises from three factors:

1. the general stem cell concept, explicated as a simple abstract model;

2. the basic design of stem cell experiments; and

3. the epistemic situation that motivates these experiments.

The first two are presented above. ${ }^{13}$ As for the third: the epistemic situation in which stem cell experiments are performed is one in which we do not know exactly which charactervalues (if any) are had by all and only stem cells from a given lineage $L$. If we did have this information, we could identify stem cells in advance using the "stem cell signature," which would replace $C$ with a specification of stem cell character-values ( $c f$. Leychkis et al 2009). But the character-values of stem cells from some lineage $L$ are what these experiments aim to discover; we do not know them in advance.

Given these three factors, consider what would be required for a stem cell experiment to identify a single cell as a stem cell. To determine its self-renewal ability, a cell is placed in an environment that inhibits differentiation, and its descendants measured. The result is a measurement of that cell's self-renewal relative to time-interval $n$ and characters $C$. To determine a single cell's differentiation potential, the cell is placed in an environment conducive to differentiation, and its descendants measured. The result is a measurement of that cell's differentiation relative to time-interval $t_{1}-t_{2}($ or $n)$ and characters $M$. It is not possible to perform both experiments on a single cell. This is because the two tests require different environments, while the initiating cell does not persist across division events in either context. (Stem cell biologists literally don't know what they've got 'til it's gone.) So there is no way to experimentally measure both self-renewal and differentiation ability for a single cell. But stem cells are defined as having both capacities. It follows that stem cells cannot be identified and characterized at the single-cell level. ${ }^{14}$ This evidential constraint does not depend on the details of particular experiments or limitations of our current technology. It arises from the general definition of 'stem cell' (explicated in terms of the abstract model above) and the basic design of stem cell experiments.

As noted, our epistemic situation is also crucial. If we knew exactly which charactervalues are had by all and only stem cells from a given lineage $L$, then this "signature" would replace variable $C$. Cells measured and shown to exhibit the stem cell signature could then be used as replicates from a homogeneous stem cell population to determine self-renewal and differentiation potential. But this is not the epistemic situation that motivates stem cell experiments. The aim is to identify and characterize stem cells; we do not know their

13 The evidential constraint could be avoided by changing either the general stem cell concept or basic experimental design. One proposal for the former is to seek experimental evidence for the molecular grounds of the property of 'stemness' rather than 'stem cells' (Leychkis et al 2009, Fagan 2013a Chapter 3). Other possibilities exist, not limited to those currently realized or proposed by scientists. A systematic exploration of these options is a task for future work. Thanks to an anonymous reviewer for THEORIA for raising this point. This paper's aim is more modest: to defend the evidential constraint in stem cell research practice.

${ }^{14}$ This is not the whole story for stem cell uncertainty, but it is the claim targeted by the objection. For more detail, see Fagan (2013a, 2013b). 
characters in advance. Given these conceptual, methodological, and epistemic factors, stem cell experiments cannot identify and characterize single stem cells.

\section{Crucial stem cell experiments}

Laplane's objection to the above is straightforward: there are, she claims, experiments that measure self-renewal and differentiation at the single-cell level. More specifically, it is possible to "perform both experiments" - that is, test for both self-renewal and differentiation - using "single cell (serial) transplantations" of putative stem cells into irradiated mice (Laplane, pers. comm. 2013). If this is correct, then some experiments can unequivocally determine whether a given single cell is a stem cell. The results of such 'crucial stem cell experiments' contradict the evidential limitation stated above.

A high-profile example of such an experiment was recently reported in Nature (2013). Shalin Naik and colleagues used viruses to individually "barcode" cells from mouse bone marrow that share a set of surface receptors. ${ }^{15}$ Cells from this population were injected into mice treated with radiation to destroy their blood and immune cells, providing 'physiological space' for transplanted cells to replenish those systems. Any immune cells that subsequently appear in the animal must therefore be derived from transplanted cells. Two weeks later, tissue from surviving mice was analyzed to trace the descendants of individually "bar-coded" cells (Figure 3). This experimental design allowed researchers to track the mature progeny of each transplanted cell and determine resulting "cell fates" - different kinds of blood and immune cells exhibiting distinct functions, morphology and molecular traits. Naik and colleagues' experiment clearly measures differentiation for single cells. Because immune cells have very short lifespans, self-renewal is required to maintain an organism's immune system over time. So the experiment also measures self-renewal, albeit for a short time-interval (two weeks). It seems to follow, then, that both self-renewal and differentiation potential can be measured by experiments of this kind: single-cell in vivo transplantation experiments. Such experiments are not minor or peripheral in stem cell biology, but serve as an exemplary "gold standard" for large areas of the field (Melton and Cowan 2009).

More precisely, single-cell in vivo transplantation experiments serve as the "gold standard" for identifying and characterizing one kind of stem cell: mouse blood-making (hematopoietic) stem cells (HSC). HSC experiments have several features that distinguish them as a variant of the generic design presented above (Section 2, Figure 2). First, as in vivo experiments, they use whole animals to measure stem cell self-renewal and differentiation potential. This contrasts with in vitro stem cell experiments, which use cell lines in artificial culture to assess self-renewal and differentiation potential. ${ }^{16}$ Laplane and others agree that the evidential constraint discussed above holds for in vitro stem cell experiments. Our dispute

15 This "cell differentiation antigen" profile was: CD $16 / \mathrm{CD} 32 / \mathrm{CD} 127 / \mathrm{CD} 117^{\mathrm{hi}} / \mathrm{Sca} 1^{+} / \mathrm{CD} 135^{\mathrm{hi}}$ (Naik et al 2013, Supplement). This profile specifies the set character-values $C$ of putative stem cells in the experiment.

16 The in vivo/in vitro distinction is drawn in various ways across biological fields. In stem cell biology, in vitro methods are those that situate cells outside of any organismal body. See Landecker (2007) for the significance of cell culture techniques more generally. 
a

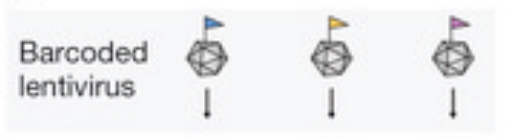

b

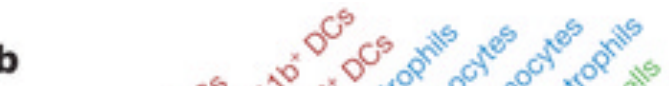

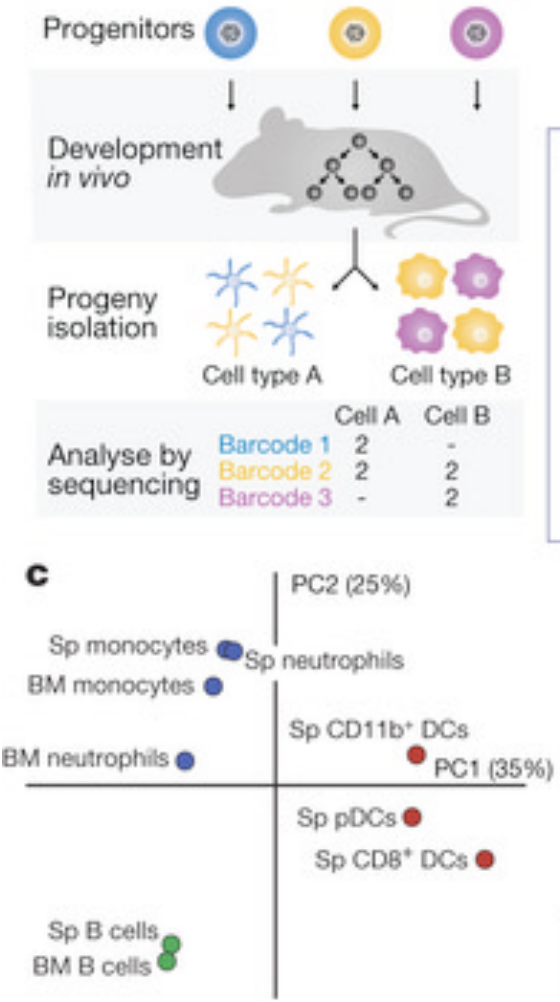

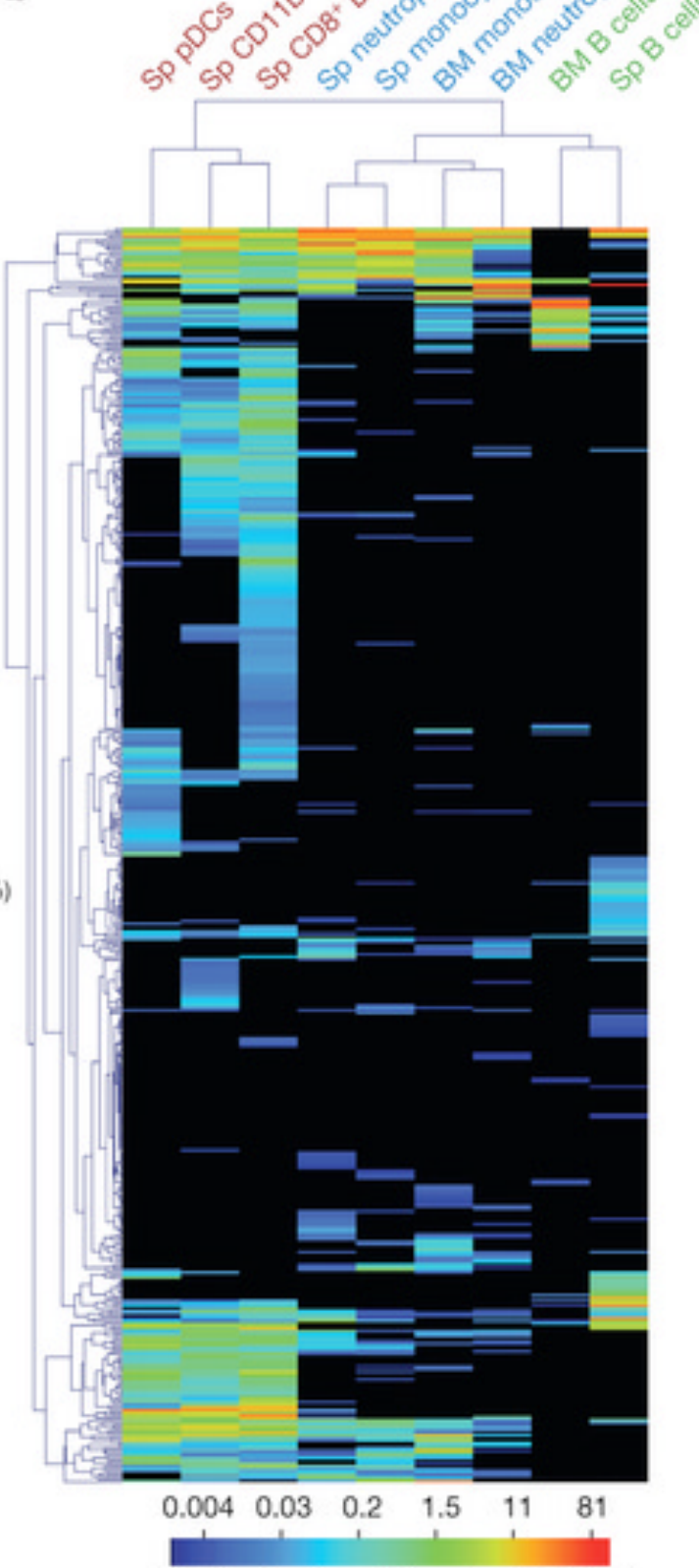

Contribution to cell type by LMPP (\%)

Figure 3. Single cell in vivo transplantation experiments: a recent example (from Naik et al. 2013, 229). Used by permission of Nature Publishing Group 
concerns only in vivo stem cell experiments. In such experiments, the 'internal milieu' of an inbred mouse serves as the environment in which self-renewal and differentiation take place. This environment is more complex, less controlled, and less accessible to observation than in vitro cell culture. But the in vivo system is not 'natural' or unmanipulated. Inbred mice are highly-engineered model organisms (Rader 2004), further intervened on by radiation to selectively destroy their blood and immune cells. Without an immune system, the mice die within a few weeks. Long-term survival (3-6 months) indicates a functioning immune system, which must be derived from transplanted cells.

A second distinctive feature of single-cell in vivo HSC transplantation experiments is that HSC are not products of cell culture, like embryonic stem cells, but already present in adult tissues - specifically, bone marrow and (to a lesser extent) the umbilical cord and circulating blood. The challenge is to find the rare HSC by subdividing bone marrow cells into populations sharing particular combinations of surface molecules. Naik et al's "barcode" innovation is a step beyond the standard single-cell method for HSC research: fluorescence-activated cell sorting (FACS), a technology akin to ink-jet printing that sort cells one-by-one into populations with shared values for characters such as size, density, and surface molecule expression. In the standard experiments, sorted cell populations are serially diluted before injection, so single cells are transplanted into mice for 'radiation rescue' - this is the "single cell (serial) transplantation" noted by Laplane. Any immune cells that subsequently appear in 'rescued' mice must be derived from a single transplanted cell. A third distinctive feature of Naik et al's experiment is that self-renewal and differentiation are measured concurrently rather than at different stages of an HSC experiment. Without an immune system, the mice die within a few weeks. Because immune cells have short lifespans, self-renewal is required to maintain the immune system over time. Survival of three to six months indicates a functioning immune system, which must be composed of differentiated descendants of the transplanted cell. So both self-renewal and differentiation are measured in the same in vivo environment, during the same time interval. This arrangement suggests a merging of the two measurements, which take place in two different environments in the basic stem cell experimental design (see above).

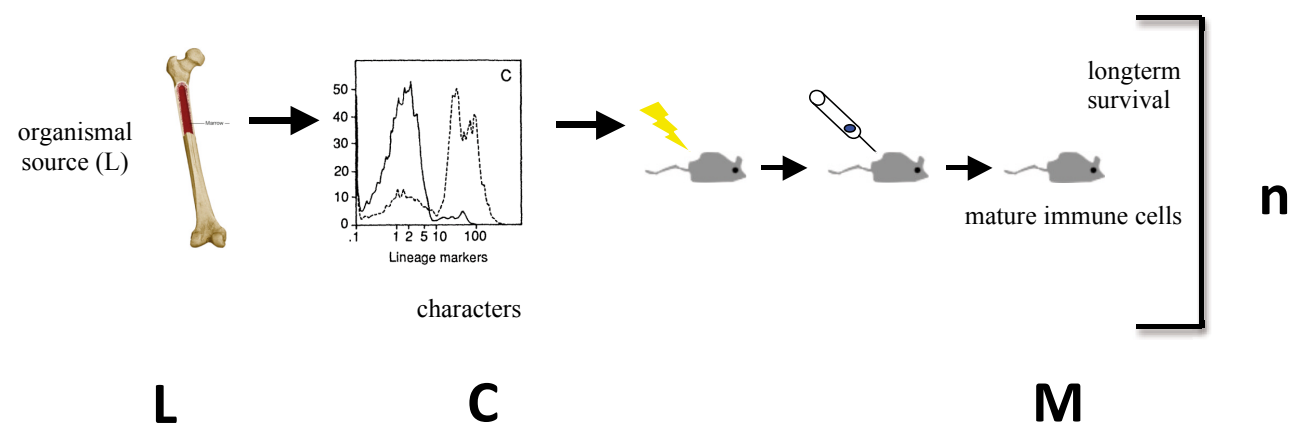

Figure 4. Crucial stem cell experiments: basic design

These features notwithstanding, HSC experiments in mice do share this same basic experimental design (Figure 4). First, bone marrow cells are collected from an inbred mouse: this 
specifies the cell lineage of interest $(L)$. Second, extracted cells are sorted into sub-populations with shared values of morphological, functional and molecular characters, including cell size, density and presence or absence of specific surface molecules. This measurement specifies $C$, the set of characters relative to which stem cell capacities are assessed. The measurement takes place in a new artificial environment, assumed to have no effect on cells' character-values (in part because this stage lasts only a few hours). Third, single cells from sorted populations are transferred to a new environment: an irradiated mouse. After several months (or weeks, for the barcode experiments) tissue samples are taken to measure differentiation ability of transplanted cells, by testing for the presence of different kinds of mature immune cells with distinct morphologies, surface receptors and functions. This final stage specifies the time interval of interest $n\left(\right.$ or $\left.t_{1}-t_{2}\right)$ and the set of characters relative to which differentiation is assessed $\left(M_{\mathrm{i}}\right)$.

Single-cell in vivo transplantation experiments of this sort fall within the scope of my model and the consequent evidential limitation. The three factors that engender the limitation all hold in this case. First, HSC conform to the general definition of a stem cell ( $\mathrm{Ta}$ ble 1), so the abstract model should apply. Second, as noted, the HSC experimental design exhibits the pattern discussed above (Figure 2). The epistemic situation that motivates these experiments is less clear-cut. Here it is important to distinguish between two experimental aims: identifying and characterizing HSC, on the one hand; and determining developmental capacities of cells assumed to be $H S C$, on the other. The evidential constraint on single stem cells concerns only the former (see Section 2). Naik et al's barcode experiments have the latter aim: the researchers assume that cells with a particular set of $C$-values are HSC, and use innovative experimental methods to determine these cells' developmental capacities at the single-cell level. Interestingly, their results suggest that HSC so defined are heterogeneous: cells that share these character-values may give rise to one, two, or more kinds of immune cell $(2013,229-230)$. The single-cell results show so much variation, in fact, that Naik and colleagues can draw no clear conclusions about developmental pathways, and instead use principal components analysis to identify population-level patterns (see Figure 3). Heterogeneous developmental capacities of cells assumed to be HSC in advance of stem cell experiments is a recurring theme in four decades of bone marrow transplantation research. ${ }^{17}$

The heterogeneity of results in Naik et al's experiments cast doubt on their assumption that HSC have been identified. Rather, identification of HSC is a work-in-progress, advancing since the first single-cell assays of the early $1960 \mathrm{~s} .{ }^{18}$ So although the intent behind Naik and colleagues' experiments puts them beyond the scope of my claim about the evidential constraints of stem cell experiments, their results indicate that these claims are still relevant. Moreover, single cell in vivo transplantation experiments that aim to identify and characterize HSC, rather than presupposing HSC already 'in hand,' are perfectly feasible, as Laplane notes. In such cases, all three conditions for the single cell evidential constraint apply. This poses a significant challenge to the account presented above: apparently crucial experiments for stem cell capacities, which unambiguously demonstrate that a single cell can both self-renew and differentiate to produce all the cells of the immune system.

17 See Fagan 2013a, Chapters 2 and 8, for detailed discussion of the history of HSC research.

18 Till and McCulloch (1961). 
Laplane's objection demands a careful response. The next section defends my original claim with a three-part rebuttal.

\section{Rebuttal}

The above sections set out both sides of the debate: the model-based account of stem cells and its evidential consequences, on the one hand; and the crucial experiments that appear to flout this constraint, on the other. In this section, I argue that the appearance of contradiction is illusory. Single-cell in vivo transplantation experiments do not violate the evidential constraints of my model-based account, for at least three reasons. I discuss each in turn.

\section{a) Self-renewal}

First, the allegedly crucial experiments do not measure self-renewal for the single transplanted cell. A single-cell transplantation into an irradiated mouse that subsequently survives with a functioning immune system shows that the 'input' of that one cell produces an 'output' of diverse immune system cells, which is maintained for several months. Self-renewal is not measured for any particular cell in the lineage, but indirectly inferred from the experiment's endpoint: generation and maintenance of a functioning immune system. Recall that self-renewal is a form of cell proliferation; a cell divides to produce at least one offspring cell with the same values for a set of characters $C$. It is difficult to imagine that all the cells of the immune system could be generated from a single originating cell without self-renewal occurring somewhere in the lineage - but Naik et al's experiment does not test that assumption. The character-values of the transplanted cell are not checked for similarity with those of its descendants. Only differentiated progeny of the transplanted cell are measured. The relevant comparisons for these are diverse immune cell types $\left(M_{\mathrm{i}}\right)$. It is possible, for all the experiment shows, that every cell division event in the lineage stemming from the transplanted cell involves changes in characters $C$, such that no self-renewal occurs.

This scenario strains credulity, however, since vast increases in cell number are required to generate the immune system and its long-term maintenance requires that short-lived cell types be repeatedly replenished. So a single-cell transplantation experiment (with this outcome) provides very strong evidence that self-renewal has occurred during the time interval of interest. Given well-established background assumptions about the 'lifespan' of different blood and immune cell types, the results of single-cell transplantation experiments show that self-renewal is very likely to have occurred. But all this tells us is that self-renewal has occurred somewhere in the lineage of which the transplanted cell is the origin. It does not show that the transplanted cell itself is capable of self-renewal, which is the point at issue. Perhaps the transplanted cell divides to produce two more specialized progenitors that are capable of self-renewal, and these in turn give rise to distinct blood cell lineages. Or self-renewal might be deferred to a pool of four, eight, or sixteen slightly-varying progenitors and so on. The single-cell transplant experiment tells us nothing about the lineage relations connecting the input of the transplanted cell with the output of specialized immune cells, measured weeks or months later. This is because the in vivo experimental design does not allow access to intermediate developmental stages. It is partly for this reason that debate about the structure of pathways of early blood cell development has persisted for decades 
(e.g., Spangrude et al 1988, Kondo 2010). Indeed, Naik et al (2013) add yet another new proposal to those already on offer, rather than settling the debate.

So self-renewal results of single-cell in vivo transplantation experiments do not conflict with my model-based account. The evidential constraint associated with that model does not assert that we can never experimentally test for self-renewal in a cell lineage. The claim, rather, is that we cannot experimentally show that a single cell -in this case, the transplanted immune-system-generating cell- is capable of both self-renewal and differentiation. ${ }^{19}$ In vivo single-cell transplant experiments can tell us that self-renewal has (very likely) occurred somewhere in the cell lineage. But they do not demonstrate that the transplanted cell (or any other single cell in the lineage) is capable of self-renewal.

\section{b) Differentiation}

Second, single-cell transplant experiments do not establish the transplanted cell's differentiation potential. Rather, they show that the transplanted cell can give rise to blood and immune cells within the body of a particular (extensively manipulated) mouse. But this result does not tell us what specialized cell types that cell might give rise to in other environmental contexts. Recall that a cell's differentiation potential is revealed by placing it in an environment conducive to differentiation, then measuring its descendants to see whether these exhibit specialized features of mature cells. Different kinds of mature cell are elicited by different environments; there is no generic environment for cell differentiation. A single cell can be placed in (at most) one such differentiation-conducive environment. We cannot realize the full range of differentiation potential from a single stem cell, if that range exceeds one mature cell type. (And if it does not, we cannot experimentally discover this.) So we cannot tell what a cell's descendants would be like in a different range of environments, or without any experimental manipulation. In addition, because transplanted cells are simply 'turned loose' in the organismal body, the HSC experimental design does not identify 'differentiation-inducing' environments within the mouse or distinguish these from environments conducive to self-renewal. The results of single-cell transplantation experiments, therefore, do not provide any basis for comparison across cell capacities across environments, which could determine the full range of a cell's differentiation potential. This is why debates about the full extent of HSC differentiation capacity (aka "adult stem cell plasticity") are difficult to settle, and why claims that HSC can be used in place of embryonic stem cells continually arise, abetted by political motivation.

As with self-renewal, single-cell in vivo transplantation experiments do tell us something about differentiation. They show that the transplanted cell has a capacity for differentiation in the organism's 'internal milieu' that extends at least to the major cell types comprising the immune system. So the experiments unambiguously identify single cells with some capacity for differentiation. They do not, however, reveal the limits of this capacity - that is, they do not fully determine that cell's differentiation potential. These transplanted single cells, as noted above, also give rise to a lineage in which self-renewal occurs. Setting aside the problems with attributing self-renewal to a single transplanted cell, one could say that these experiments identify single cells with some capacity for differentiation and self-renewal. But

19 For further argument against experimental evidence for self-renewing stem cells, see Fagan (2013a, 2013b). 
this is not the same as identifying and characterizing stem cells. The category of cells with some capacity for differentiation and self-renewal is much more inclusive than the category of cells currently recognized as stem cells. The former includes, arguably, all cells that appear during the course of development, except for 'terminally differentiated' cells. The term 'stem cell' is used in a more restricted way, to designate cells that are 'origins' of a cell lineage, with maximal differentiation and self-renewal capacities (see Table 1). It is the latter concept the abstract stem cell model aims to explicate. Different kinds of stem cell are defined as exhibiting differentiation potential varying from a single mature cell type to all the cell types comprising the adult organism. So if we cannot identify a single cell's differentiation potential, then we cannot classify it in the way stem cells are classified in practice. Single-cell in vivo transplantation experiments do not overcome the evidential constraint, because a single cell's differentiation potential cannot be determined by one transplant experiment. To determine the full range of developmental possibilities, we would need to test a population of identical 'replicate' cells in a wide range of environments.

\section{c) Circularity}

The idea of a population of 'replicate' stem cells suggests a way to experimentally determine a single cell's differentiation potential, bypassing at least this aspect of the evidential constraint. Suppose that we have a population of candidate stem cells that are identical, or homogeneous, qua stem cell; i.e., all cells in the population have the same developmental capacities. Given this homogeneous stem cell population, we can determine the differentiation potential of a single transplanted cell with a set of replicate single-cell experiments that systematically vary the environment of cell differentiation. ${ }^{20}$ This approach seems to bypass the evidential constraint I defended above, at least as regards differentiation potential.

However, experiments like Naik et al's cannot assume a homogeneous population of stem cells at the outset, if the experiment aims to identify and characterize stem cells of that population. Given that experimental aim, the assumption of a homogeneous stem cell population involves an epistemic circularity. Single-cell transplants are drawn from a population of candidate stem cells that are homogeneous with respect to the values for characters $C$ that are measured during the experiment. For example, Naik and colleagues sorted cells from mouse bone marrow to identify a population with the surface profile CD16/CD32-/ CD $127^{-} / \mathrm{CD} 117^{\mathrm{hi}} / \mathrm{Sca} 1^{+} / \mathrm{CD} 135^{\mathrm{lo}} / \mathrm{CD} 150^{+}$(Naik et al 2013, Supplement). For any such experiment, some set of character-values defines the population of candidate stem cells, which is therefore homogeneous with respect to those characters. But it does not follow that cells of that population are identical, or homogeneous, qua stem cell. That is what the experiment aims to discover. We do not know in advance that those character-values are the right ones for identifying stem cells for the lineage in question - or even if $C$ is the right set of characters. A method using a different character-set $C$ might very well pick out a different cell population to transplant. ${ }^{21}$

20 Although the in vivo 'internal milieu' is too coarse-grained to yield such systematic results.

21 Indeed, different laboratories and research groups often use different characters $C$, or associate different $C$-values with 'stemness.' This is one reason that claims about stem cells should be understood as restricted, or relative, to particular experimental contexts. 
The epistemic situation is challenging, because we have no way other than experiment to identify stem cells' properties and capacities. There is no predetermined list of characters and character-values that pick out all and only stem cells; experimental researchers must work this out for themselves. This is done via many iterations of the experimental design sketched above, which relates cell character-values to developmental capacities for self-renewal and differentiation (Figure 2). But, for the reasons given above, this two-stage experimental design cannot identify stem cells at the single-cell level. So we cannot show that all the cells in a particular population are individual stem cells with the same capacities for self-renewal and differentiation. That is, we cannot experimentally demonstrate that a population of candidate stem cells is in fact a homogeneous population of stem cells. For experiments aiming to identify stem cells, the assumption of stem cell homogeneity is unavoidably provisional.

As noted above, many experimental reports of in vivo single-cell transplantation experiments (including Naik et al 2013) presuppose that the transplanted cells are a homogeneous population of blood-making stem cells (HSC). This practice is unproblematic, if the assumption is understood as provisional - a working hypothesis, subject to revision. Experimental results indicating developmental heterogeneity within the population (such as those of Naik et al) prompt revision of a homogeneity hypothesis, leading experimenters to seek further characters to distinguish different sub-populations ("sub-sets") within the original candidate stem cell population. ${ }^{22}$ In this way, stem cell experiments proceed via a 'falsifying' pattern: a proposed set of candidate stem cells with character-values $C$ is found to exhibit developmental heterogeneity, prompting a revised (expanded) set of character-values.

The homogeneity assumption for stem cells is also unproblematic if the experiment is an application of prior knowledge, rather than an effort to identify stem cells. If we were in an epistemic position to know the character-values shared by all and only mouse HSC, then we could isolate that cell population from mouse bone marrow and transplant identical single stem cells into different environments (in vivo or in vitro). This effectively treats members of the HSC population as replicates of a single cell, overcoming the restriction on determining a single cell's differentiation capacity discussed above. However, if we were in this epistemic position, we would already have HSC 'in hand.' There would be no need to perform an experiment to identify and characterize HSC. We could simply apply our knowledge of HSC to isolate all and only these cells for medical use. ${ }^{23}$ This case falls outside the scope of the evidential constraint on identifying single stem cells (see Section 2).

A problem arises, however, if these two unproblematic situations are conflated; i.e., the homogeneity assumption is not treated as a provisional hypothesis subject to revision, but presumed true in the interpretation of experimental results, as though we already had a "stem cell signature" in hand. In this situation, the assumption of a homogeneous stem cell population involves a form of epistemic circularity. And this is just the assumption required for allegedly crucial stem cell experiments to determine the full range of a single stem cell's differentiation potential through 'replicate' experiments. Crucial stem cell experiments are

22 This is the course followed by the history of HSC research: a steadily-lengthening list of character-values used to identify this type of stem cell (see Fagan 2013a, Chapter 8).

23 This is the protocol for bone marrow transplantation using HSC - but that procedure involves identifying stem cell capacities in populations, not at the single-cell level. 
not applications of prior knowledge. ${ }^{24}$ They claim to identify and characterize stem cells at the single-cell level; that is, to show that a single cell with measured character-values $C$ has maximal self-renewal and differentiation capacity (measured in terms of $n$ and $M_{\mathrm{i}}$ ) within lineage $L$. But, for the reasons given above, self-renewal and differentiation capacities of a single cell are not measured by in vivo transplantation experiments. The constraint on measuring differentiation, at least, is lifted if it is assumed that the experiment is performed on a homogeneous population of stem cells, which can furnish replicate single cells. But how can that assumption be justified? Our only access to stem cells is via experiments, and those that identify and characterize stem cells share the basic design that gives rise to the evidential constraint at issue. So the homogeneity assumption can be justified only if we are able to identify and characterize stem cells of some lineage $L$ before performing the experiment.

This is the circularity problem: to interpret the results of a stem cell experiment as 'crucial' (identifying HSC at the single-cell level) is to presuppose the experiment's success. A successful stem cell experiment measures characters $C$, values of which identify all and only stem cells with particular developmental capacities (relative to some values of $n$ and $M_{\mathrm{i}}$ in lineage $L$ ). If we have a homogeneous population of stem cells with which to assay the differentiation capacity of single cells, then success of the experiment is a foregone conclusion. To put the point another way, for an experiment to reveal the differentiation (and self-renewal) capacity of a single transplanted cell requires an assumption that renders the experiment itself pointless. So the circularity here is not harmless. The claim of a crucial stem cell experiment relies on a presumption that can be taken as true only if we are in an epistemic situation that undercuts the motivation for the experiment itself. This epistemic circularity problem blocks what appears to be a reasonable way to avoid the evidential constraint on experimental identification of single stem cells.

These three considerations rebut Laplane's objection. The allegedly crucial HSC experiments show that a single cell has some differentiation capacity and self-renewal in a lineage. But this does not conflict with the evidential constraint arising from the abstract stem cell model and the basic design of stem cell experiments (together with the epistemic situation in which those experiments are performed). My statement of this limitation asserts that experiments aimed at identifying stem cells and their capacities cannot demonstrate those capacities for a single cell. The contrary position, for the experiments at issue, commits an epistemic circularity. I conclude that single-cell in vivo transplantation experiments like those of Naik and colleagues do not overcome the evidential constraint on identifying single stem cells.

\section{Source of dispute}

I now turn to the source of the dispute. Laplane's objection, also raised by several stem cell researchers, is motivated by commitment to the epistemic value of stem cell experiments.

${ }^{24}$ Although, as noted above, Naik et al's experiment can be interpreted in this way - in which case my claims about evidential constraints do not apply to that case. The question arises, however, of how our knowledge of HSC was acquired in the first place, and for those experiments the uncertainty arguments come back into play (see also Fagan 2013b). 
The evidential constraints I propose as part of the model-based approach appear to challenge this commitment, placing limits on our ability to experimentally detect stem cell capacities. This could be seen as encouraging skepticism about single stem cells, or even stem cells in general. From this perspective, a defense of stem cell experiments' epistemic value is called for. But in fact, experimentalists and I are in agreement here. The purpose of my abstract model, and consequent claims about stem cell uncertainty, is not to prompt skepticism about stem cell capacities, but to facilitate methodological caution and clarity. This section further clarifies my model-based approach to stem cell research, showing how the allegedly crucial stem cell experiments fit into and support this viewpoint. The next section considers features of experimental practice that present obstacles to this 'compatibilist' stance.

The abstract model is a philosophical intervention in stem cell research - it is not a representation that scientists themselves construct or use. The model's purpose is not to describe or predict experimental methods or results, but to explicate the concept that unifies diverse stem cell research projects and their variety of experimental entities. To return to Giere's formulation (see Section 2), I use the abstract stem cell model to represent the field's central concept, for the purpose of finding some unity amidst the bewildering diversity of stem cell research practices. The purpose of that unification, in turn, is to render stem cell research more accessible to non-experts; i.e., nearly everyone apart from stem cell biologists themselves. So the abstract model as such does not make 'direct contact' with stem cell experiments, and is not intended as a tool for stem cell researchers (except perhaps in efforts at outreach).

The evidential constraint on identifying single stem cells is a consequence of applying the abstract model to experimental methods and results. The model is applied to particular experiments by using the details of materials and methods to specify its variables. It is applied to the whole class of stem cell experiments (in the sense specified in Section 2) by noting the ways these variables are specified in the basic experimental design. The evidential constraint follows from the latter application of the abstract model to stem cell experiments, given further assumptions about our epistemic situation vis-à-vis stem cells. One way to understand this philosophical project is in hypothetico-deductive terms, as a 'metaexperimental' prediction; i.e., a prediction about experimental evidence, which is derived from my abstract model and experimental conditions. On this view, the allegedly crucial stem cell experiments appear to be disconfirming observations, and so a good test of my model. The arguments in Section 4 can be interpreted in hypothetico-deductive terms as well: showing that there is no contradiction between the prediction (claims about the evidential limitations of stem cell experiments) and observations from experimental practice. ${ }^{25}$ While not entirely out of place, this hypothetico-deductive gloss of my project exemplifies now-familiar limitations of traditional theories of scientific inquiry: restricting the role of experiment, reducing its diverse scientific aims and activities to hypothesis-testing. The relation of my abstract stem cell model to experimental practices in stem cell research is not best articulated in terms of hypothesis-testing, with the evidential constraint playing the role of a prediction to be challenged by experiment. Instead, my claim about evidential lim-

25 That the observations in this case are claims about experimental evidence is just the 'meta' aspect of this interpretation. 
itations of stem cell experiments is better conceived as the starting-point for a more finegrained and nuanced account of stem cell research. Such a construal fits well with a modelbased view of scientific inquiry, emphasizing the "familial" relations linking more abstract with more concrete models (e.g., Giere 2004).

On this model-based approach, the evidential constraint defended above clarifies a number of important features of stem cell research today, including the field's standard for experimental success. For over 50 years, stem cell biologists have looked to experimental techniques to manage uncertainties that follow from the stem cell concept. The most prevalent response is to adopt what I term "the single-cell standard" (Fagan 2013a, b). This stance takes as its experimental ideal a single cell in a controlled environment, with all relevant 'signals' taken into account, so results reflect all and only the reproductive output of that single starting cell. Measured stem cell capacities can then be unambiguously attributed to that cell in that environment. Technologies that track a single cell's reproductive output over time, combined with techniques that measure character values of single cells, can yield data of this sort. Progress in stem cell biology takes the form of more detailed and direct measurements of single cells and their descendants.

The single-cell transplant experiments, far from contradicting my view, are precisely in line with the general trend that is explicated by the evidential constraints I propose. By improving our 'grasp' on single candidate stem cells, single-cell transplant experiments provide better evidence for stem cell capacities, compared to earlier methods using cell populations. None of this contradicts the in-principle evidential constraint on identifying single stem cells. Experiments that satisfy the single-cell standard provide evidence about stem cell capacities - but only relative to the set of characters and environments used in the experiment. Inferences that go beyond these experimental situations are unavoidably speculative. More general results are obtained from replicate experiments using a range of environments, given the (provisional) assumption that the starting population is homogeneous. But as new cell characters $C$ and $M$ are discovered or made accessible to measurement, and new environments engineered, the homogeneity assumption must be continually reassessed and revised.

The evidential constraint defended above is therefore not an indictment of experimental practices in stem cell biology, nor a challenge to the epistemic value of stem cell experiments. Rather, it articulates a constraint within which these experiments operate, thereby making intelligible a number of general features of stem cell research. These include, as noted above, long-running debates over developmental potential of stem cells from different sources, and the endurance of the single-cell standard. Another feature of the field, rapid turnover in stem cell hypotheses and terminology, is also explained: due to the evidential constraint, substantive models of stem cells are provisional and rapidly become obsolete. The evidential constraint also helps us understand the significance of the variety of stem cell types (e.g., Table 1). This diversity is not a symptom of incomplete understanding, but a consequence of the general stem cell concept. Claims about stem cells based on different elaborations of the basic model do not conflict. The diversity of stem cells should not be a source of contention, but a positive resource for inquiry. Finally, this account of experiment evidence in stem cell research challenges the traditional dichotomy of science and technology. Technical innovations that increase experimenters' ability to measure and track single cells provide stronger evidence for hypotheses about stem cells, by more closely approximating the ideal single-cell standard. Single-cell technologies 
are thus an important form of progress in stem cell biology, with evidential significance. Instead of progress through refinements of theories and models, stem cell biology makes progress through technological innovations that improve our access to developmental activities of single cells.

The single-cell in vivo transplantation experiments cited by Laplane and others are not counterexamples to the model-based account, but rather instances of the single-cell standard at work in the stem cell field. These apparently crucial experiments are part of a more general trend that my model-based account motivates and clarifies. There is always some uncertainty about the stem cell capacities we can measure, for single stem cells. But this is ground for methodological caution, not full-blown skepticism about stem cell capacities. So the evidential constraint defended in the above sections is not an attack on the epistemic value of stem cell experiments, but part of a general framework that vindicates them within the wider context of the field.

\section{Experimental assumptions}

We can now see the basic tension driving this debate. The abstract stem cell model presented above is a theoretical construct. It selects and explicates what I take to be essential features of the stem cell concept. This model and its experimental applications 'cover' a wide range of stem cell phenomena. My approach therefore adds something to the field that does not fully comport with stem cell researchers' own epistemic and explanatory norms. The latter are, understandably, closely tied to standards of experimental progress, which hinge on technical improvements in our 'grasp' of single cells. It is reasonable that stem cell biologists, and philosophers who work closely with them and adopt their perspective, would respond to my claims as though they were directly about experiments. Experimental results and their interpretation are the currency of stem cell researchers' own arguments.

The hypothetico-deductive model of scientific inquiry may also be implicated here. This traditional account, though offering a limited and reductive view of experimentation, enjoys wide currency in many scientific fields today. As discussed above, my argument in Section 2 above may be understood in hypothetico-deductive terms, as a secondorder prediction requiring meta-experimental test. Experimental scientists accustomed to thinking of their own inquiries in hypothetico-deductive terms may implicitly use the same framework to assess my account of stem cell research. In this framework, progress is made through potentially disconfirming experimental tests. The counterexample proposed by Laplane and others appears to be a good, rigorous test of my view (Section 3); my rebuttal in Section 4 can also be glossed in these terms. But, as the previous section argues, the hypothetico-deductive formulation misses the point of my project. The evidential constraint and the abstract model that underlies it are intended to explicate the background conceptual framework within which stem cell experiments take place.

There may be good reasons for experimental biologists to ignore such background considerations. The challenge of innovating and troubleshooting experimental methods to reveal new aspects of complex biological systems is formidable. Stem cell biologists spend considerable resources of time, energy, and funds in generating experimental results. So it is understandable that they tend to see those results as the 'main event' of scientific inquiry, 
rather than as fragments of a broader view. From this local standpoint, the conceptual background that is the primary focus of my account may well appear otiose or irrelevant. In its indirect relation to the fine-grained details of experimental methods and results, my model-based account resembles the covering-law view of explanation: a philosophical artifact "peripheral" to the actual practices of experimental biology (Schaffner 1993). From a perspective immersed in experimental methods and results, the apparent contradiction between crucial stem cell experiments and the claims about stem cell uncertainty may be my account's only point of interest.

Are there considerations to counterbalance experimenters' resistance to abstract interventions by philosophers? Although full treatment of this issue is beyond the scope of this paper, the stem cell debate suggests several avenues to pursue in future work. A modelbased approach to inquiry, with tolerance for multiple purposes, allows for more options than traditional methodology. Experimental biologists, particularly in the stem cell field, are well aware of the importance of outreach to broader audiences. Even the most narrowly focused, insular projects benefit from finding ways to extend experimental techniques and results to other contexts. Model-based accounts that aim to engage non-expert audiences, like the abstract stem cell model and its consequences, could be valuable tools in these 'extra-laboratory' aspects of experimental practice. Another way in which abstract modeling approaches can contribute to experimental inquiry is in helping keep track of the epistemic status of key background assumptions presupposed by particular experiments. Many stem cell experiments, including that of Naik et al (2013), proceed from the assumption that some population of stem cells (such as HSC) are already 'in hand,' identified and characterized. This provisional working hypothesis is easily confused with robust scientific fact, leading to an inflated view of our epistemic situation vis-à-vis stem cells. ${ }^{26}$ The experimental literature on stem cells leads to the impression that we already know, at least for some cases, a set of character-values $C$ had by all and only stem cells. However, cross-experimental comparisons of results indicate (independently of the arguments presented here) that no such set of character-values exists for stem cells in general (Leychkis et al 2009). By focusing attention on exactly what is assumed in generating a particular experimental result, against a background framework including the abstract definition of stem cells, a model-based approach can help prevent 'premature optimism' in stem cell research. This in turn could counteract the tendency to over-hype the prospects for this field, offering a more realistic picture for audiences outside the community of stem cell researchers.

\section{Conclusion}

Contra Laplane and several stem cell scientists, I have argued that experiments cannot identify and characterize single stem cells, because we cannot directly measure self-renewal and differentiation for a single cell. The single-cell transplantation experiments cited by my critics do not overcome this evidential constraint, for at least three reasons. First, the experiments do not measure self-renewal for a single cell. Second, these in vivo experiments

\footnotetext{
26 See Section $4 c$ above.
} 
do not establish the precise differentiation potential for a single cell. Third, their results interpreted in terms of single cells presuppose facts not yet known - the very facts about stem cells that are under experimental investigation. The experiments do show that some degree of self-renewal and differentiation occurs following a successful single-cell transplant. But this does not unequivocally establish that the transplanted cell is itself a stem cell. The single-cell transplant experiments are not counterexamples to the evidential constraint I have defended, but well-motivated responses to it, conforming to the 'single-cell standard' that marks progress in stem cell research. In addition to this defense of my view, I have traced the roots of the dispute to contrasts between my own abstract modeling approach, and the aims and standards of experimentalists working in that field. Finally, I offer some suggestions for overcoming these differences of perspective.

\section{REFERENCES}

Fagan, M.B. 2013a. Philosophy of Stem Cell Biology. London: Palgrave Macmillan.

Fagan, M.B. 2013b. The stem cell uncertainty principle. Philosophy of Science 80: 945-57.

Fagan, M.B. forthcoming. Cell and body: individuals in stem cell biology. To appear in Individuals Across Sciences, eds., T. Pradeu \& A. Guay. London: Oxford University Press.

Giere, R. 1988. Explaining science: A Cognitive Approach. Chicago: University of Chicago Press.

Giere, R. 2004. How models are used to represent reality. Philosophy of Science 71: 742-52.

Gottweis, H., B. Salter \& C. Waldby. 2009. The Global Politics of Human Embryonic Stem Cell Science. Palgrave McMillan.

Kondo, M. ed. 2010. Hematopoietic Stem Cell Biology. New York: Humana Press.

Landecker, H. 2007. Culturing Life. Cambridge: Harvard University Press.

Laplane, L. 2013. Cancer stem cells: ontology and therapies. Ph.D. Dissertation, Sorbonne Université. To be published by Harvard University Press.

Leychkis, Y., S. Munzer \& J. Richardson. 2009. What is stemness? Studies in History and Philosophy of Biological and Biomedical Sciences 40: 312-320.

Maienschein, J. 2003. Whose View of Life? Cambridge: Harvard University Press.

Melton, D.A. \& C. Cowan. 2009. Stemness: definitions, criteria, and standards. In Essentials of Stem Biology, $2^{\text {nd }}$ edition, eds. Robert Lanza et al., xxii-xxix. San Diego, CA: Academic Press.

Mazzolini, R.G. \& H-J. Rheinberger. 2012. Differing Routes to Stem Cell Research: Germany and Italy. Bologna: Il Mulino; Berlin: Duncker \& Humblot.

Naik, S.H. et al. 2013. Diverse and heritable lineage imprinting of early haematopoietic progenitors. Nature 496: 229-32.

Nobel Foundation. 2012. The 2012 Nobel Prize in Physiology or Medicine - Press Release. Nobelprize.org. 10 Oct 2012, http://www.nobelprize.org/nobel_prizes/medicine/laureates/2012/press.html

Rader, K. 2004. Making Mice. Princeton: Princeton University Press.

Ramalho-Santos, M. \& H. Willenbring. 2007. On the origin of the term 'stem cell.' Cell Stem Cell 1: 35-38.

Schaffner, K. 1993. Discovery and Explanation in Biology and Medicine. Chicago: University of Chicago Press.

Spangrude, G., S. Heimfeld \& I.L. Weissman. 1988. Purification and characterization of mouse hematopoietic stem cells. Science 241: 58-62.

Takahashi, K. \& S. Yamanaka. 2006. Induction of pluripotent stem cells from mouse embryonic and adult fibroblast cultures by defined factors. Cell 126: 663-76.

Takahashi, K., K. Tanabe, M. Ohnuki, M. Narita, T. Ichisaka, K. Tomoda \& S. Yamanaka. 2007. Induction of pluripotent stem cells from adult human fibroblasts by defined factors. Cell 131: 861-72. 
Thompson, C. 2013. Good Science: the Ethical Choreography of Stem Cell Research. Cambridge: MIT Press. Thomson, J., J. Itskovitz-Eldor, S. Shapiro, M. Waknitz, J. Swiergel \& J. Jones. 1998. Embryonic stem cell lines derived from human blastocysts. Science 282: 1145-47.

Till, J.E. \& E.A. McCulloch. 1961. A direct measurement of the radiation sensitivity of normal mouse bone marrow cells. Radiation Research 14: 213-22.

Melinda Bonnie Fagan is Associate Professor of Philosophy at the University of Utah, where she holds the Sterling McMurrin Chair. Her research focuses on experimental practice in biology (particularly stem cell and developmental biology), explanation, and modeling. She is the author of Philosophy of Stem Cell Biology (Palgrave Macmillan, 2013) and more than twenty-five articles and book chapters on topics in philosophy of science and biology.

Address: The University of Utah, 215 South Central Campus Drive, Carolyn Tanner Irish Humanities Building, Salt Lake City, UT 84112, USA. E-mail: mel.fagan@utah.edu 